\title{
Itinerário terapêutico: tendência em teses e dissertações da enfermagem no Brasil
}

\author{
Dalva Cezar da Silva1 Maria de Lourdes Denardin Budó ${ }^{1}$ Maria Denise Schimith ${ }^{3}$ Salete de Jesus Souza Rizzatti'Bruna \\ Sodré Simon ${ }^{4}$ Marianne Lopes Robaina ${ }^{5}$
}

\begin{abstract}
RESUMO
Objetivou-se identificar a tendência acerca do itinerário terapêutico em teses e dissertações defendidas em programas de pós-graduação em Enfermagem do Brasil. Trata-se de uma revisão narrativa, realizada no Banco de Teses e Dissertações da Coordenação de Aperfeiçoamento Pessoal de Nível Superior. Para coleta de dados utilizou-se o termo itinerário terapêutico, como assunto, com a opção todas as palavras e sem recorte temporal. Encontrou-se 84 estudos, dos quais foram selecionados 17 resumos. A partir da análise dos resumos identificaram-se os temas: Itinerário terapêutico de famílias e de pessoas com condições crônicas de saúde; Aspectos relacionados ao surgimento e manejo da doença; Relação profissional-usuário e utilização dos serviços de saúde; e ltinerário terapêutico e Enfermagem. 0 conhecimento sobre o itinerário terapêutico possibilita uma prática de enfermagem mais próxima à realidade dos usuários dos serviços de saúde. Verificou-se a tendência qualitativa e com enfoque socioantropológico.
\end{abstract}

Descritores: Enfermagem; Aceitação pelo paciente de cuidados de saúde; Cuidados de enfermagem; Doença crônica; Cultura.

\section{Therapeutic itinerary: trend in nursing theses and dissertation}

\begin{abstract}
It aimed to identify the trend about therapeutic itinerary in theses and dissertations presented in Nursing graduate programs of the Brazil. This is a narrative review realized in the Theses and Dissertations Database of Coordenação de Aperfeiçoamento Pessoal de Nível Superior. In the data collection was used the term therapeutic itinerary, as subject, with option all words and out time period. It was found 84 studies which 17 abstracts were selected. From the analysis was identified the issues: Therapeutic itinerary of families and people with chronic health conditions; Aspects related to the emergence and disease management; Professional-user relationship and use of the health services; and Therapeutic itinerary and Nursing. The knowledge about the therapeutic itinerary enables a nursing practice closer to the reality of users of the health services. There was a qualitative trend and focusing socioanthropological.
\end{abstract}

Descriptors: Nursing; Patient acceptance of health care; Nursing care; Chronic disease; Culture.

\footnotetext{
${ }^{1}$ Mestre em enfermagem pela Universidade Federal de Santa Maria (UFSM), Santa Maria, RS, Brasil.

${ }^{2}$ Doutora em enfermagem pela Universidade Federal de Santa Catarina (UFSC), Florianópolis, SC, Brasil.

${ }^{3}$ Doutora em ciências pela Universidade Federal de São Paulo (UNIFESP), São Paulo, SP, Brasil.

${ }^{4}$ Mestranda em enfermagem na Universidade Federal de Santa Maria (UFSM), Santa Maria, RS, Brasil.

${ }^{5}$ Graduanda em enfermagem na Universidade Federal de Santa Maria (UFSM), Santa Maria, RS, Brasil.
} 


\section{Introdução}

No Brasil, a procura de respostas para os problemas de saúde faz com que as pessoas peregrinem pelo sistema de saúde. Isso em parte pode ser justificado, pois a busca por cuidados de saúde, nem sempre ocorre conforme o fluxo de atendimento preconizado pelo Sistema Único de Saúde (SUS). Além disso, na relação profissional-usuário pode haver a influência do modelo biomédico, o qual geralmente valoriza as questões biológicas e desvaloriza as, referentes ao contexto sociocultural' ${ }^{1}$.

O cuidado inicia no contexto em que a pessoa vive. As pessoas articulam crenças e práticas sobre a saúde e a doença existentes no seu contexto cultural, com a reinterpretação das práticas médicas oficiais².

Dessa forma, é preciso compreender as experiências construídas pelas pessoas no processo de adoecimento, o que irá orientar as escolhas sobre os cuidados e os tratamentos que irão realizar, ou seja, o itinerário terapêutico. 0 percurso que as pessoas fazem em busca de cuidados e tratamentos de saúde é chamado de itinerário terapêutico. A enfermagem pode utilizar esse saber como uma estratégia para o cuidado, ao conhecer as possibilidades encontradas pelas pessoas ou famílias para resolver seus problemas de saúde 3 .

A enfermagem tem se voltado para o enfoque no SUS e da valorização do contexto e saber da pessoa cuidada, durante a formação acadêmica. De maneira semelhante, o conhecimento construído na pós-graduação stricto sensu, desempenha um papel fundamental e reconhecido politicamente, na formação de recursos humanos qualificados.

Assim, justifica-se a importância do levantamento da produção científica realizada na Pós-graduação stricto sensu, pois algumas vezes essa ainda não se encontra divulgada no formato de artigos científicos publicados ${ }^{4}$.

Frente a isso, questiona-se: qual a tendência acerca do itinerário terapêutico em teses e dissertações defendidas nos programas de pós-graduação em Enfermagem (PPGENF) do Brasil? A partir desse questionamento, objetivou-se identificar a tendência acerca do itinerário terapêutico em teses e dissertações defendidas PPGENF do Brasil.

\section{Metodologia}

Revisão narrativa, pesquisa bibliográfica ampla sob o "estado da arte", adequada para descrever e discutir 0 desenvolvimento de um determinado assunto, sob ponto de vista teórico ou contextual. ${ }^{5}$

A busca foi desenvolvida no Banco de Teses da Coordenação de Aperfeiçoamento de Pessoal de Nível Superior (CAPES). A coleta dos dados ocorreu em outubro de 2012, utilizando-se o termo itinerário terapêutico como assunto, com a opção todas as palavras e sem delimitar um recorte temporal. Inicialmente foram localizados 84 estudos, incluindo 15 teses, 64 dissertações de mestrado acadêmico e cinco de mestrado profissional. Destes, procedeu-se a leitura dos títulos e resumos para a seleção.

Utilizou-se, como critério de inclusão, o fato de os trabalhos serem da temática itinerário terapêutico; ser pesquisa desenvolvida por enfermeiros em (PPGENF) do Brasil e ter resumo disponível online e gratuitamente. Excluíram-se as teses ou dissertações com resumos incompletos ou que abordassem a busca por cuidados dentro de mesma uma instituição de saúde.

Foram selecionados e analisados qualitativamente, por meio da análise dos temas, os resumos de 17 estudos, dos quais três teses e 14 dissertações de mestrado acadêmico. Todas as informações obtidas neste estudo foram extraídas, somente dos resumos disponibilizados no banco de teses da CAPES.

Para a descrição das produções selecionadas, elaborou-se um quadro sinóptico, com as seguintes informações: tipo (tese ou dissertação); ano de publicação; instituição de origem; região; cenário e sujeitos do estudo; abordagem metodológica (quali-quanti, qualitativa ou quantitativa); referencial teórico-filosófico e metodológico e os desfechos, para posterior análise, que inter-relacionada com a literatura disponível fortaleceu a discussão dos resultados.

A análise dos dados ocorreu quantitativamente e qualitativamente, por meio da aproximação de temas comuns nas teses e dissertações, ou seja, as informações semelhantes foram agrupadas em uma mesma categoria. 


\section{Resultados}

O itinerário terapêutico foi estudado em cinco universidades do Brasil. Quanto à região e à instituição, destacase a Região Sul com oito produções, sendo seis da Universidade Federal de Santa Catarina (UFSC); uma da Universidade Federal do Paraná (UFPR) e uma da Universidade Estadual de Maringá (UEM). Na Região CentroOeste oito, em que todas as produções estão vinculas à Universidade Federal de Mato Grosso (UFMT). Na Região Sudeste uma, da Universidade Federal do Rio de Janeiro (UFRJ), conforme destaca o quadro a seguir:

Quadro 1 - Teses e Dissertações de enfermagem brasileira sobre itinerário terapêutico - Banco de Teses da CAPES, 2012.

\begin{tabular}{|c|c|c|c|}
\hline Tipo & Ano & Título & Universidade \\
\hline T & 2011 & $\begin{array}{l}\text { A magnitude da tuberculose e os itinerários terapêuticos dos Munduruku do Pará } \\
\text { na Amazônia brasileira }{ }^{5}\end{array}$ & UFRJ \\
\hline T & 2010 & $\begin{array}{l}\text { Tecendo os fios da saúde mental em Belém/PA: visibilidades ou possibilidades da } \\
\text { rede de cuidado } 6\end{array}$ & UFSC \\
\hline $\mathrm{T}$ & 2009 & O Centro Espírita no itinerário terapêutico em situações de vida ${ }^{7}$ & UFSC \\
\hline D & 2011 & $\begin{array}{l}\text { Vivência da condição crônica por adrenoleucodistrofia de criança e família: possi- } \\
\text { bilidades da mediação jurídica na garantia do direito à saúde }{ }^{8}\end{array}$ & UFMT \\
\hline D & 2011 & $\begin{array}{l}\text { Mediação nas redes para o cuidado à saúde na experiência de adoecimento por } \\
\text { condição crônica decorrente do câncer colorretal }{ }^{9}\end{array}$ & UFMT \\
\hline D & 2010 & Câncer infantil: a experiência em família ${ }^{10}$ & UFMT \\
\hline D & 2010 & O significado da experiência do adoecimento por câncer: um estudo biográfico ${ }^{11}$ & UFMT \\
\hline D & 2009 & O Itinerário terapêutico das famílias de crianças com diarreia ${ }^{12}$ & UFSC \\
\hline D & 2008 & O itinerário terapêutico: história oral de idosos com câncer ${ }^{13}$ & UFPR \\
\hline D & 2008 & $\begin{array}{l}\text { As práticas e o direito à saúde: a vivência de uma mulher com câncer do colo do } \\
\text { útero }^{14}\end{array}$ & UFMT \\
\hline D & 2008 & $\begin{array}{l}\text { Práticas regulatórias no SUS: um estudo de caso sobre a regulação para a emer- } \\
\text { gência cardiocirculatória de um morador de São José do Rio Claro/MT }{ }^{15}\end{array}$ & UFMT \\
\hline D & 2008 & $\begin{array}{l}\text { A experiência da enfermidade e o itinerário terapêutico de uma pessoa com } \\
\text { complicações cardiovasculares procedente de Marcelândia - } \text { MT }^{16}\end{array}$ & UFMT \\
\hline D & 2007 & $\begin{array}{l}\text { O cuidado de Enfermagem no itinerário terapêutico da pessoa com diagnóstico } \\
\text { de câncer }{ }^{17}\end{array}$ & UFSC \\
\hline D & 2007 & As necessidades em saúde de indivíduos adultos em Porto Rico-PR ${ }^{18}$ & UEM \\
\hline D & 2007 & $\begin{array}{l}\text { A experiência de adoecimento e a busca por cuidado empreendida pela pessoa } \\
\text { com Diabetes Mellitus }^{19}\end{array}$ & UFMT \\
\hline D & 2005 & O itinerário terapêutico de indivíduos portadores de HIVIAIDS20 & UFSC \\
\hline $\mathrm{D}$ & 2004 & $\begin{array}{l}\text { O itinerário terapêutico do adolescente com Diabetes Mellitus tipo I e seus } \\
\text { familiares }^{21}\end{array}$ & UFSC \\
\hline
\end{tabular}

Fonte: Banco de Teses da CAPES, 2012.

Em relação ao ano da produção, contabilizou-se uma em 2004 e em 2005; duas em 2009; três em 2007, 2010 e 2011; e quatro em 2008.

Quanto à abordagem dos estudos, encontrou-se um quali-quantitativo6 e 16 qualitativos. ${ }^{7-22}$

Identificou-se que dos 17 estudos, nove tiveram enfoque socioantropológico, sendo utilizado o suporte teórico de Arthur Kleinman ${ }^{14,21-2}$; Arthur Kleinman e Geertz ${ }^{7-8}$; Arthur Kleinman e Madeleine Leininger ${ }^{13}$; Arthur Kleinman, Hildegard Peplau e Dorothéa Orem ${ }^{18}$; Madeleine Leininger e Luc Botanski ${ }^{19}$ e um que não apontou o teórico utilizado. ${ }^{11}$

Como participantes dos estudos encontraram-se famílias com crianças $^{9,11,13}$; adolescentes e seus familiares ${ }^{22}$; uma pessoa com câncer colorretal e sua família no contexto do SUS ${ }^{10}$; pessoas com diagnóstico de câncer e as enfermeiras da Central de Quimioterapia ${ }^{18}$; pessoas em tratamento oncológico ${ }^{12,14-5}$; indígenas ${ }^{6}$; pessoas com HIV/ 
AIDS $^{21}$; uma única pessoa em busca de atendimento. ${ }^{16-7,20}$ Pessoas com sofrimento psíquico, bem como familiares e profissionais ${ }^{7}$; adultos residentes na zona urbana do município de Porto Rico - Paraná ${ }^{19}$; pessoas que buscaram as práticas terapêuticas desenvolvidas em um Centro Espírita de orientação kardecista. ${ }^{8}$

Entre as situações de adoecimento destaca-se 0 câncer $^{10-2,14-5,18} ; 0$ diabetes mellitus ${ }^{20,22}$; as complicações cardíacas $^{16-7}$; a tuberculose ${ }^{6}$; a HIVIAIDS ${ }^{21}$; o sofrimento psíquico ${ }^{7}$; a diarreia ${ }^{13}$; a adrenoleucodistrofia ${ }^{9}$ e em dois estudos não foi constatada uma situação específica de adoecimento. ${ }^{8,19}$

Encontraram-se como temas comuns nas teses e dissertações da enfermagem brasileiras: Itinerário terapêutico de famílias e pessoas com condições crônicas de saúde; Aspectos relacionados ao surgimento e manejo da doença; Relação profissional-usuário e utilização de serviços de saúde; Itinerário terapêutico e Enfermagem.

\section{Itinerário terapêutico de famílias e pessoas com condições crônicas de saúde}

No itinerário terapêutico de famílias e pessoas com condições crônicas de saúde, contatou-se que geralmente são usados recursos de cuidado dos subsistemas familiar, popular e profissional, sendo esses recursos produzidos de acordo com o conhecimento da família, da rede de relações, e consulta em livros e materiais educativos. Também se utiliza informações adquiridas pelos meios de comunicação, da busca pela religiosidade e curadores populares. ${ }^{21}$

A busca por cuidados e tratamentos parte do subsistema familiar, depois simultaneamente do popular e do profissional, com prevalência de um ou de outro em determinados momentos. Assim, o percurso é composto de vários momentos, não sendo linear. ${ }^{22}$ Além disso, a terapêutica popular encontra-se de forma complementar e não excludente à médica, pois se identifica que diante do diagnóstico médico é mantido o uso paralelo de práticas baseadas no conhecimento e nas crenças. ${ }^{14}$

De forma semelhante, é importante o reconhecimento das aproximações e distanciamentos das relações estabelecidas pelo usuário e familiar. ${ }^{7}$ A família no itinerário terapêutico, além de geralmente ser a base para o cuidado em situações de adoecimento, ao ter um de seus integrantes com condições crônicas, sofre influência dessa situação. Isso pode ocorrer devido à necessidade de novos rearranjos na estrutura familiar. ${ }^{9}$

As trajetórias de busca, produção e gerenciamento de cuidado na experiência de adoecimento são empreendidas pela pessoa adoecida e sua família para obter resolução das necessidades em saúde prolongadas, decorrentes da condição crônica. Assim, encontra-se um percurso repleto de variados caminhos, não somente os institucionalizados pelo SUS, sendo 0 cuidado buscado em diferentes lugares. ${ }^{10}$

Nos casos de pouca resolutividade e integralidade das práticas no sistema formal de cuidados, as redes se tornam mais necessárias frente às vulnerabilidades das pessoas. ${ }^{10}$ Novos arranjos nos itinerários em busca de cuidados à saúde são influenciados pelo apoio que os usuários do SUS encontram em redes formais e informais de ajuda. A rede informal age de maneira dinâmica no que se refere à busca pelo cuidado, pois possibilita mudanças nas decisões. ${ }^{16}$

No itinerário terapêutico pode haver o trâmite pelo sistema privado de desembolso direto, conveniado e público, sendo assim, um empreendimento realizado para buscar, produzir e gerenciar os cuidados de saúde. ${ }^{12}$

\section{Aspectos relacionados ao surgimento e manejo da doença}

A identificação de um comprometimento da saúde ocorre conforme a compreensão da pessoa envolvida no evento, a qual pode entendê-la como uma situação de doença ou não. Esse reconhecimento influencia a busca por cuidados e tratamentos. Assim, percebe-se o problema, caracteriza-o, desenvolve uma ação e depois avalia os seus resultados. ${ }^{13}$

Nesse processo, questões de ordem econômica e fatores socioculturais estão presentes em todos os momentos, juntamente com a rede social..$^{13}$ De maneira semelhante, vários fatores contribuem para a entrada e a saída de um subsistema de saúde, tais como: acessibilidade, custo/benefício, eficácia, experiências anteriores, valores, crenças e a maneira como são ouvidos pelas pessoas que integram cada um dos subsistemas. ${ }^{22}$

Outro aspecto, relacionado ao surgimento e manejo da doença é o preconceito e o estigma de determinadas doenças, o que pode favorecer o isolamento social e diminuir as chances de recuperação e reinserção da pessoa acometida ao meio social. ${ }^{7,21}$

Diante da manifestação da doença, são as mulheres que, reconhecem e valorizam mais rapidamente os sinais e sintomas, buscando mais precocemente soluções junto ao sistema oficial de saúde. ${ }^{19} \mathrm{O}$ recurso mais utilizado no itinerário terapêutico é a automedicação, com pouca diferença entre os sexos. ${ }^{19}$ 


\section{Relação profissional-usuário e utilização dos serviços de saúde}

Frente às exigências de cuidado, as práticas dos profissionais de saúde são ainda pouco potentes, ao não se articularem à família na produção do cuidado.9 Encontram-se aproximações e distanciamentos das relações estabelecidas pelo usuário e familiar e profissionais/serviços de saúde. Os serviços e práticas profissionais não estão organizados para proteger o usuário ou garantir as respostas necessárias as suas demandas, pautadas no acesso à rede de cuidado, nos direitos e valores relacionados à defesa da vida humana. ${ }^{7}$

O acesso a diversos procedimentos envolvidos no cuidado pode ser dificultado pela demora do diagnóstico e tratamento, bem como pela carência de equipes multiprofissionais atuando nas Unidades de Saúde. ${ }^{7}$ Também se encontra que as formas de organização dos serviços pode ocasionar o diagnóstico tardio e que, no entanto, são as práticas de saúde desenvolvidas pelos diferentes profissionais as responsáveis pela evolução da doença, trazendo sofrimento ao usuário, além do aumento desnecessário nos custos para o SUS. ${ }^{15}$

A insatisfação com os serviços ofertados pelo sistema oficial de saúde decorre do horário disponibilizado para 0 atendimento, a reduzida oferta de consulta para atender à demanda, a dificuldade no agendamento de consultas e exames, inexistência de especialidades médicas, dificuldade na aquisição de medicamentos, baixa credibilidade no serviço médico oferecido e problemas no relacionamento com os profissionais. Assim, é preciso a adaptação dos serviços às necessidades de seus usuários e adequação da atuação da equipe de saúde..$^{19}$

Por outro lado, entre as razões para a escolha do serviço de emergência está a qualidade do atendimento prestado pelos profissionais de saúde, a falta de profissionais nas unidades de saúde, o marketing do hospital e a capacidade desses em realizar exames por diferentes motivos. ${ }^{13}$

Os vários encaminhamentos, os deslocamentos para exames e procedimentos, demoras e as desarticulações entre os serviços, são marcantes no cuidado profissional. As práticas caracterizam-se por ações prescritivas e impositivas que desconsideram as reais necessidades indivíduo. ${ }^{17}$

Assim, faz-se necessário um atendimento sob a perspectiva da integralidade e do direito à saúde, diferente da assistência centrada na atenção médica, com ênfase na doença. ${ }^{7}$ Para tanto, pontua-se incrementar a participação social dos usuários para diminuir a relação assimétrica e hierárquica entre estes e profissionais de saúde. ${ }^{15}$

Da mesma maneira, entende-se que não é possível construir práticas profissionais cuidativas que desconsiderem a experiência cotidiana de vida e de saúde da pessoa a ser cuidada, assim como não é possível propor tais práticas sem que haja a participação ativa dessa pessoa. ${ }^{20}$ Também se pode concretizar um cuidado mais sensível ao possibilitar a abertura para outras formas de cuidado, com diálogo entre as diferentes áreas de conhecimento e saberes. ${ }^{7}$

Frente a isso, os profissionais e gestores de saúde precisam compreender a subjetividade da pessoa que vivencia o processo de adoecimento, proporcionando um cuidado ético, e neste sentido, alcançar a tão almejada integralidade da atenção em saúde, por meio de ações como a escuta atenta entre pessoas adoecidas e os profissionais de saúde. ${ }^{17}$

Portanto, há vários elementos para se repensar no cuidado, desde a formação do profissional até seu preparo permanente na consideração da cidadania da pessoa e sua família na condição de saúde. ${ }^{12}$

\section{Itinerário terapêutico e Enfermagem}

A compreensão do itinerário terapêutico proporciona aos profissionais de saúde reconhecer a multiplicidade de saberes e modos de se lidar com a enfermidade, fornecendo subsídios para a construção de um cuidado de saúde mais próximo da realidade sociocultural do individuo. Além disso, percebe-se a trajetória individual perpassando o caráter público da cultura e, sobretudo, a posição da pessoa como sujeito de seu próprio cuidado. ${ }^{21}$ Dessa forma, compreender o itinerário contribuiu para uma atuação profissional mais convergente à situação vivenciada pelas pessoas. ${ }^{22}$

$O$ desenho do itinerário terapêutico se mostra como importante ferramenta para avaliar a qualidade dos serviços de saúde, bem como possibilitar detectar e corrigir falhas nas formas de organização dos serviços..$^{15} \mathrm{O}$ itinerário terapêutico pode ser um instrumento de compreensão dos recursos sociais disponíveis à produção e gerenciamento do cuidado. ${ }^{7}$

O enfermeiro foi considerado o profissional de referência para a assistência à saúde, ao descrever os itinerários terapêuticos realizados por indígenas em busca de tratamento da tuberculose. ${ }^{6}$ As características da formação de enfermagem possibilitam sua atuação ao elaborar estratégias de cuidados na perspectiva da pessoa adoecida e sua família, de modo efetivo e com práticas que sejam eficazes na vida dessas pessoas. ${ }^{9}$ 
A constituição de novas práticas de cuidados em enfermagem podem ter como base as narrativas de vida na experiência de adoecimento. ${ }^{12}$ Frente a isso, a enfermagem pode planejar cuidados na realidade sociocultural das pessoas e suas famílias ao conhecer o itinerário terapêutico percorrido por esses. ${ }^{14}$

$\mathrm{Na}$ área de enfermagem e oncologia pediátrica, encontrou-se que as práticas atuais de cuidado podem ser ampliadas ao utilizar o itinerário terapêutico como recurso que possibilita visualizar a criança no contexto familiar. ${ }^{12}$ Por outro lado, a falta de padronização e realização da sistematização dos cuidados de enfermagem de forma empírica entre os pares, favorece com que esses cuidados sejam incorporados parcialmente ao itinerário terapêutico da pessoa com diagnóstico de câncer. Para que ocorra a incorporação da sistematização dos cuidados de Enfermagem como filosofia de cuidado e para o cuidado, pontua-se a educação permanente como estratégia. ${ }^{18}$

\section{Discussão}

Encontrou-se a concentração da produção nas regiões Sul e Centro-Oeste, com predomínio de estudos qualitativos, destacando-se principalmente, o enfoque socioantropológico. Esses dados apontam a ampliação da Pós-graduação stricto sensu em Enfermagem no Brasil, pois os primeiros cursos foram implantados na região Sudeste e Sul, sendo iniciada formação de mestres na década de 1970 e de doutores a partir dos anos $80 .{ }^{23}$

As discussões sobre itinerário terapêutico concentram-se nas produções realizadas no período de 2004 a 2011. Tal fato é pontuado na literatura ${ }^{24}$, em decorrência da implantação de modelos assistenciais mais próximos à realidade local das pessoas, sendo assim, é crescente o interesse em conhecer padrões de comportamento em busca de cuidados terapêuticos e adequar práticas dos profissionais de saúde. ${ }^{24}$

Nas teses e dissertações, identificou-se a influência dos conceitos desenvolvidos por Kleinman, os quais ocupam na literatura um importante papel de referência na explicação de comportamentos de procura por cuidados e dos itinerários percorridos. ${ }^{24}$

Dessa maneira, conhecer o itinerário terapêutico permite ao profissional de saúde compreender o contexto em que o paciente se encontra, identificando suas potencialidades e suas carências. Além disso, possibilita reconhecer a repercussão da prática profissional de cuidado, assim como de outros sistemas. ${ }^{25}$

Um saber que deve ser considerado pelos profissionais como essencial no processo educativo é a experiência de quem vive com uma condição crônica. ${ }^{26}$ Ainda mais que no atendimento a essas pessoas faz-se necessário que os profissionais de saúde entendam o significado do adoecimento e atuem integrando o saber científico com o do senso comum. ${ }^{27}$

Além disso, percebe-se que na situação do adoecimento crônico a família busca elaborar estratégias que possibilitem dar conta dos sentimentos e problemas que acometem a todos integrantes. Para isso, há a organização da família, de acordo com suas possibilidades financeiras, materiais, emocionais, bem como, a influência da rede social de apoio. 0 ato de interagir com outras pessoas, faz com que o cuidado ao familiar possa ser compartilhado, a tristeza do processo crônico seja diminuída neste momento difícil. ${ }^{28}$

O cotidiano representa o cenário de base para o estudo dos itinerários terapêuticos, pois os tratamentos das doenças são construções individuais e sociais que se materializam nos jogos sociais. ${ }^{29}$ Frente a isso, as concepções sobre saúde e as atitudes na busca pela atenção à saúde podem ser influenciadas pelas experiências individuais e sociais. Nesse sentido, a experiência da doença leva os indivíduos a buscarem uma alternativa para o alívio de suas dores ou sofrimento. ${ }^{30}$

O sistema de saúde deve prover formas de facilitar o acesso e respeitar as particularidades e individualidades uma vez que existem diferenças entre os sexos na maneira de reconhecer a doença e buscar soluções. ${ }^{30}$

Ao revisar os conceitos e as ideias relacionados à saúde e à doença, encontra-se que o modelo biomédico ainda é predominante. Faz-se necessário investir em novo modelo no qual o desafio seja promover a saúde, fortalecer a autonomia dos sujeitos com respeito às diferenças, potencializar as capacidades individuais, sociais e coletivas e considerar o contexto em que as pessoas vivem. Assim, o profissional necessita compreender a saúde em suas diferentes dimensões, com o enfoque voltado para a promoção e proteção da mesma, e considerar as práticas integradoras, as desigualdades sociais que impedem o acesso aos serviços de saúde e à informação por parte da população como um todo. ${ }^{31}$ 
Na relação com os profissionais da saúde, identifica-se geralmente a visão reducionista e limitada do processo saúde-doença que exclui os fatores históricos, sociais e culturais. Deste modo, entre esses e as pessoas cuidadas, forma-se um distanciamento marcado pela concepção da doença como somente biológica, o que difere do significado da experiência vivenciada pela pessoa, a qual possui seu conhecimento adquirido ao longo do caminho percorrido até chegar ao profissional. ${ }^{25}$

Conhecer as diferentes práticas socioculturais permite ao profissional de saúde compreender a maneira de pensar e agir dos indivíduos frente as suas aflições, facilitando a comunicação entre eles, possibilitando um cuidado coerente e favorecendo as intervenções. ${ }^{32}$

Nesse sentido, o enfermeiro como importante membro da equipe de saúde, ao implementar cuidados que aproximam os saberes populares e os científicos, visa na sua prática, uma assistência integral que compreenda 0 contexto cultural do indivíduo e da família. Por consequência, isso permite a promoção da saúde e a melhoria da qualidade de vida. ${ }^{1}$ Para que isso aconteça é fundamental que os profissionais da saúde repensem suas práticas de maneira crítica, a fim de que suas ações tornem-se resolutivas no decorrer do adoecimento crônico e não imediatistas, realizadas de modo não programático com a situação vivenciada por cada indivíduo e sua família. ${ }^{33}$

Portanto, evidencia-se a pertinência dos estudos sobre itinerários, tanto para a reflexão sobre as relações entre profissional de saúde e paciente, quanto para as atividades de planejamento e gestão. A gestão em saúde, ao apresentar desenhos assistenciais centrados no usuário e em seu território, encontra o desafio de conhecer as características e os determinantes da busca de cuidado. Para isso, vislumbra-se a contribuição da abordagem socioantropológica a partir de uma perspectiva ampliada que associa a percepção do paciente à análise do contexto real em que se inscrevem as suas práticas de cuidado. ${ }^{24}$

\section{Conclusão}

Este revisão oportunizou identificar à tendência das dissertações e teses desenvolvidas sobre itinerário terapêutico em Programas de Pós-graduação stricto sensu em Enfermagem, no Brasil. Constatou-se que a maioria dos estudos adotou a metodologia qualitativa e a abordagem socioantropológica. As produções sobre itinerário terapêutico concentram-se nos Programas de Pós-graduação em Enfermagem das regiões sul e centro-oeste do Brasil.

A realização desse estudo permitiu caracterizar o conhecimento sobre itinerário terapêutico, o qual pode contribuir para as práticas em enfermagem. Esse saber possibilita ao profissional um olhar que favorece um processo de reflexão sobre a realidade da pessoa cuidada. Além disso, contribui para a proposição do enfermeiro, ofertar soluções adequadas ao contexto em que as práticas em saúde se concretizam.

A partir do levantamento das dissertações e teses de enfermagem sobre itinerário terapêutico foi possível encontrar que os temas abordados reportam-se para a problemática das famílias e pessoas com condições crônicas de saúde; questões referentes ao gerenciamento da doença; relação entre profissional-usuário e utilização dos serviços de saúde; e por fim Itinerário terapêutico e Enfermagem. Tem-se como fator de limitação do estudo, a dificuldade de análise dos dados de alguns resumos, devido falta de clareza na apresentação dos achados. Porém o levantamento foi válido para a divulgação do panorama da produção do conhecimento stricto sensu da enfermagem brasileira e para apontar a possibilidade de novos estudos.

\section{Referências Bibliográficas}

1. CeolinT, Heck RM, Barbieri RL, Schwartz E, Muniz RM, Pillon CN. Plantas medicinais: transmissão do conhecimento nas famílias de agricultores de base ecológica no Sul do RS. Rev esc enferm USP. 2011;45(1):47-54.

2. Melo LP, Silva NP, Silva KCL, Ponte MPTR, Gualda DMR. Representações e práticas de cuidado com a ferida crônica de membro inferior: uma perspectiva antropológica. Cogitare enferm. 2011;16(2):303-10.

3. Mattosinho MMS, Silva DMGV. Itinerário terapêutico do adolescente com diabetes mellitus tipo I e seus familiares. Rev Latino-Am Enfermagem. 2007;15(6):1113-9.

4. Garcia RP, Budó DML, Barbosa MS, Simon BS, Leal TC, Oliveira SG. Caracterização das teses e dissertações de enfermagem acerca do infarto do miocárdio. Revista Saúde (Santa Maria). [periódico na Internet]. 2012[citado 2013 mar 16];38(2):67-82. Disponível em: http://cascavel.ufsm.br/revistas/ojs-2.2.2/index.php/revistasaude/article/view/6310 
5. Rother ET. Revisão sistemática X Revisão narrativa. Acta Paul Enferm. 2007;20(2):vi.

6. Nogueira LMV. A magnitude da tuberculose e os itinerários terapêuticos dos Munduruku do Pará na Amazônia brasileira. Rio de Janeiro: [s.n.], 2011. 162 p. Tese (Doutorado) - Universidade Federal do Rio de Janeiro.

7. Pereira LS. Tecendo os fios da saúde mental em Belém/PA: visibilidades ou possibilidades da rede de cuidado. Florianópolis: [s.n.]; 2010. 184 p. Tese (Doutorado) - Universidade Federal de Santa Catarina.

8. Fenili RM. O Centro Espírita no itinerário terapêutico em situações de vida. Florianópolis: [s.n.], 2009. 130 p. Tese (Doutorado) - Universidade Federal de Santa Catarina.

9. Nepomuceno MAS. Vivência da condição crônica por adrenoleucodistrofia de criança e família: possibilidades da mediação jurídica na garantia do direito à saúde. Cuiabá: [s. n.], 2011. 155 p. Dissertação (Mestrado) - Universidade Federal de Mato Grosso.

10. Mufato LF. Mediação nas redes para o cuidado à saúde na experiência de adoecimento por condição crônica decorrente do câncer colorretal. Cuiabá: [s.n.], 2011. 167 p. Dissertação (Mestrado) - Universidade Federal de Mato Grosso.

11. Navarro JP. Câncer infantil: a experiência em família. Cuiabá: [s.n.], 2010.133 p. Dissertação (Mestrado) - Universidade Federal de Mato Grosso.

12. Medeiros LHL. O significado da experiência do adoecimento por câncer: um estudo biográfico. Cuiabá: [s.n.], 2010. 119 p. Dissertação (Mestrado) - Universidade Federal de Mato Grosso.

13. Souza AC. O Itinerário terapêutico das famílias de crianças com diarreia. Florianópolis: [s.n.], 2009. 123 p. Dissertação (Mestrado) - Universidade Federal de Santa Catarina.

14. Visentin A. O itinerário terapêutico: história oral de idosos com câncer. Curitiba: [s.n.], 2008. 146 p. Dissertação (Mestrado) - Universidade Federal do Paraná.

15. Villa MCE. As práticas e o direito à saúde: a vivência de uma mulher com câncer do colo do útero. Cuiabá: [s.n.], 2008. 153 p. Dissertação (Mestrado) - Universidade Federal de Mato Grosso.

16. Santos EJF. Práticas regulatórias no SUS: um estudo de caso sobre a regulação para a emergência cardiocirculatória de um morador de São José do Rio Claro/MT. Cuiabá: [s.n.], 2008. 130 p. Dissertação (Mestrado) - Universidade Federal de Mato Grosso.

17. Nabão FRZ. Aexperiência da enfermidade e o itinerário terapêutico de uma pessoa com complicações cardiovasculares procedente de Marcelândia - MT. Cuiabá: [s.n.], 2008. 152 p. Dissertação (Mestrado) - Universidade Federal de Mato Grosso.

18. Rosa LM. O cuidado de enfermagem no itinerário terapêutico da pessoa com diagnóstico de câncer. Florianópolis: [s.n.], 2007. 111 p. Dissertação (Mestrado) - Universidade Federal de Santa Catarina.

19. Merino MFGL. As necessidades em saúde de indivíduos adultos em Porto Rico-PR. Maringá: [s.n.], 2007. 109 p. Dissertação (Mestrado) - Universidade Estadual de Maringá.

20. Faria APS. A experiência de adoecimento e a busca por cuidado empreendida pela pessoa com diabetes mellitus. Cuiabá: [s.n.], 2007. 224 p. Dissertação (Mestrado) - Universidade Federal de Mato Grosso.

21. Malinska ICA. O itinerário terapêutico de indivíduos portadores de HIVIAIDS. Florianópolis: [s.n.], 2005. 155 p. Dissertação (Mestrado) - Universidade Federal de Santa Catarina.

22. Mattosinho MMS. O itinerário terapêutico do adolescente com diabetes mellitus tipo 1 e seus familiares. Florianópolis: [s.n.], 2004. 127 p. Dissertação (Mestrado) - Universidade Federal de Santa Catarina.

23. Erdmann AL, Fernandes JD. Publicações científicas qualificadas na enfermagem brasileira. Rev bras Enferm. 2009; 62(4):499-01.

24. Cabral ALLV, Vianna ALL, Martinez-Hemáez A, Andrade EIG, Cherchiglia ML. Itinerários terapêuticos: 0 estado da arte da produção científica no Brasil. Ciênc. saúde coletiva. [periódico na Internet]. 2011[citado 2013 mar 16]; 16(11):4433-42. Disponível em: http://www.scielo.br/scielo.php?script=sci_arttext\&pid=S1413-81232011001200016

25. Maliska ICA, Padilha MICS. AIDS: a experiência da doença e a construção do itinerário terapêutico. Rev Eletr Enf. [periódico na Internet]. 2007[citado 2013 mar 16];9(3):687-99. Disponível em: http://www.fen.ufg.br/revista/v9/n3/ v9n3a09.htm

26. Boell JEW, Meirelles BHS, Silva DMGV, Lessmann JC.. Arterial hypertension and diabetes mellitus: health care in a basic unit. J Nurs UFPE on line [periódico na Internet]. 2012 [citado 2013 mar 26];6(6):1485-90. Disponível em: http:/l www.revista.ufpe.br/revistaenfermagem/index.php/revista/article/view/2209 
27. Favoreto CAO, Cabral CC. Narrativas sobre o processo saúde-doença: experiências em grupos operativos de educação em saúde. Interface. 2009;13(28):7-18.

28. Silveira CL, Budó MLD, Ressel LB, Oliveira SG, Simon BS. Apoio social como possibilidade de sobrevivência: percepção de cuidadores familiares em uma comunidade remanescente de quilombos. Ciênc. cuid. saúde. [periódico na Internet]. 2011[citado 2013 mar 16];10(3):585-92. Disponível em: http://periodicos.uem.br/ojs/index.php/ CiencCuidSaude/article/view/17190/pdf

29. Leite AN, Vasconcellos MPC. Negociando fronteiras entre culturas, doenças e tratamentos no cotidiano familiar. Hist Cienc Saúde-Manguinhos. [periódico na Internet]. 2006 [citado 2013 mai 29];13(1). Disponível em: http://www.scielo.br/scielo.php?pid=50104-59702006000100007\&script=sci_arttex.

30. Merino MFGL, Marcon SS. Concepções de saúde e itinerário terapêutico adotado por adultos de um município de pequeno porte. Rev Bras Enferm. 2007;60(6):651-8.

31. Backes MTS, Rosa LM, Fernandes GCM, Becker SG, Meirelles BHS, Santos SMA. Conceitos de saúde e doença ao longo da história sob o olhar epidemiológico e antropológico. Rev enferm UERJ. [periódico na Internet]. 2009 [citado 2013 mar 29]; 17(1):111-7. Disponível em: http://www.facenf.uerj.br/v17n1/v17n1a21.pdf

32. Rosa LM, Silva AMF, Pereima RSMR, Santos SMA, Meirelles BHS. Família, cultura e práticas de saúde: um estudo bibliométrico. Rev enferm UERJ. [periódico na Internet]. 2009[citado 2013 mar 29];17(4):516-20. Disponível em: $<$ <ttp://www.facenf.uerj.br/v17n4/v17n4a11.pdf

33. Garcia RP, Budó MLD, Simon BS, Wünsch S, Schimith MD, Oliveira SG. Cotidiano e aprendizado de cuidadores familiares de doentes crônicos. Ciênc cuid saúde. 2011;10(4):690-6.

\section{Dalva Cezar da Silva}

Endereço para correspondência - Av. Roraima, nº 1000, Cidade Universitária. Bairro Camobi, CEP: 97105 900. Santa Maria, RS, Brasil.

Maria de Lourdes D Budó - lourdesdenardin@gmail.com

Maria Denise Schimith - ma.denise2011@gmail.com

Salete de Jesus S Rizzatti - saleterizzatti@gmail.com

Bruna Sodré Simon - enf.brusimon@gmail.com

Marianne Lopes Robaina - mari.robaina@hotmail.com

Recebido em 06 de junho de 2013.

Publicado em 14 de novembro de 2013. 
